EMBRYARIDDLE
Aeronautical University

SCHOLARLY COMMONS
International Journal of Aviation, Aeronautics, and Aerospace

$11-21-2016$

\title{
SMS Derived vs. Public Perceived Risk in Aviation Technology Acceptance (Literature Review)
}

Paul L. Myers III

Embry-Riddle Aeronautical University, myersiip@erau.edu

Follow this and additional works at: https://commons.erau.edu/ijaaa

Part of the Cognition and Perception Commons

\section{Scholarly Commons Citation}

Myers, P. L. (2016). SMS Derived vs. Public Perceived Risk in Aviation Technology Acceptance (Literature Review). International Journal of Aviation, Aeronautics, and Aerospace, 3(4). https://doi.org/10.15394/ ijaaa.2016.1141

This Literature Review is brought to you for free and open access by the Journals at Scholarly Commons. It has been accepted for inclusion in International Journal of Aviation, Aeronautics, and Aerospace by an authorized administrator of Scholarly Commons. For more information, please contact commons@erau.edu. 
The National Aeronautics and Space Administration (NASA), major airlines, and similar organizations that utilize a Safety Management System (SMS) are periodically implementing aviation technology changes. Typically, SMS derived and public perceived risk comprise two different processes with dissimilar influencing factors between the two processes. Organizational or public perceived risk level is the attitude derived from examining possible hazards and the probability of those hazards occurring to the organization, individual, equipment, society, or environment. The disparity between the organizational and public processes often causes public technology acceptance reluctance or rejection. Thus, the purpose of this paper is to identify and examine the processes and influencing factors that cause this disparity to better facilitate future aviation technology implementation.

There have been numerous research studies examining technology acceptance with risk perception research roots going back to the 1960s to research the reasons for public reluctance or rejection (Sjöberg, 2000). Some examples of risk perception studies include the Wildavsky, \& Dake (1990) study of theories of risk perception, the Whitfield, Rosa, Dan, \& Dietz (2009) study of perceptions related to nuclear power and the Townsend (2013) study of the perception of risk in the public sector. Gupta, Fischer and Frewer (2012) reviewed 292 technology acceptance studies between 1977 and 2008 finding 31 determinant factors that influenced technology acceptance. Of the 31 factors, perceived risk was the most frequently occurring variable and the one that was investigated the most in those studies. Clothier, Greer, Greer and Mehta (2015) and La Porte and Metlay (1975a) also agree on the significance of perceived risk when compared to other factors. Dobbie and Brown (2014) echo the sentiment that perceived risk is a hindrance in technology acceptance. In their study of an attempted implementation of an integrated Australian urban water management approach, they found some similarities in determining factors between practitioners and the public. However, the two distinct evaluation processes of those factors resulted in different perceptions of risk between the two groups and created a public risk-averse culture (Dobbie \& Brown, 2014).

Aviation technology innovation has progressed from the first flight of the Wright Brothers to landing on the moon in just 63 years (Lawrence, 2014). However, disparity between the SMS and public processes coupled with dissimilar influencing factors has, at times, slowed or halted technology implementation. There are several historical examples to illustrate this. One example is Robert Goddard, a rocket pioneer, who in the mid-1930s developed a rocket with movable vanes and rudders. The U.S. government, a consolidated voice of public sentiment, had no interest until it was realized that the Germans had a sizable lead in rocket 
development when they became a threat some 20 years later during WWII (Lawrence, 2014). More recently, both chemical and nuclear technologies have evoked large public perceived risk assessments, essentially creating progress stagnation. Because of this stagnation, it has been difficult to find landfills, incinerators, and other chemical facility sites and virtually impossible to dispose of the wastes from chemical and nuclear operations and processes much to the dismay of technical experts (Slovic, 1997). More specifically, nuclear waste disposal has been a 30-year struggle, largely due to the public perception as being victims rather than beneficiaries of the technology. This resulted in a lack of trust in the government and an increased perceived risk by the public. As a result, progress implementing the Yucca Mountain project, the only proposed nuclear waste site, has been stopped, hence thwarting the expansion of nuclear power production (Slovic, Layman, \& Flynn, 1991).

Future technologies requiring technology implementation and; therefore, acceptance continue to be explored such as NASA's movement toward a one-pilot airline cockpit. Even today, NASA realizes the effect of perceived risk, identifying it as one of three significant barriers to success (Warwick, 2013).

\section{The Problem}

The problem is twofold. First, SMS organizations, when implementing technology, use a reactive versus a proactive approach. Second, they do not grasp the magnitude of the public's perceived risk due to lack knowledge of the public perceived risk derivation process and influencing factors. These two elements cause a disparity between organizational and public perceived risk levels resulting in technology implementation being slowed or halted. More specifically, the introduction of new technology involves some level of SMS defined risk to the public that is viewed as acceptable by the organization. However, the public may perceive the SMS defined risk at a more negative level than the implementing organization using SMS; thus, creating a disparity (Hunter, 2001). While perceived risk is recognized to some degree as an influencing factor in technology acceptance in today's society, technology implementation is attempted concurrently with addressing public perceived risk in a reactive versus proactive approach. This reactive approach coupled with not grasping the magnitude of the impact of negative public perceived risk on technology acceptance has resulted in undesired end-states. By understanding SMS derived versus the public risk assessment processes and associated influencing factors of each, and if necessary, the methodology to change that perception once formed, public perceived risk can be targeted for either elimination or minimization to facilitate technology implementation. Additionally, using a proactive type approach, some public 
support predictability can be achieved when implementing new technology. Thus, toward this goal, SMS and public risk derivation processes, major SMS and public perceived risk influencing factors, along with the ability to influence public perceived risk once an attitude is formed, are examined in a literature review with conclusions and recommendations formed.

A literature review process was used to summarize previous studies, inform the reader of the current status of the subject area, to identify relations, contradictions, and gaps in the literature, and finally to suggest the next research steps. The process used to derive the information for the literature review was conducted using the Embry-Riddle Aeronautical University Hunt Library databases and Google Scholar by using public perceived risk and perceived risk with technology acceptance in the search function. Initially, using public perceived risk in the search criteria yielded a total of 1,158 results from the Hunt Library databases. After a quick scan of the sources, the subject matter was surmised to be too broad in scope, because of non-relevant subject areas such as food, etc. After the search criteria were narrowed to include technology acceptance in the search function and peer reviewed selected as criteria, the results were narrowed to 738 sources. Sources were then screened to omit those that could not be obtained, those that did not relate to technology acceptance or perceived risk, and those that did not include aviation, nuclear, information technology, or other potentially controversial technical areas. Google Scholar initially yielded 705 results, but the subject areas were too broad in scope, because of non-relevant subject matter, similar to what the Hunt Library search results yielded. After technology acceptance had been included in the search criteria, the search results yielded the final sources needed for the literature review.

\section{Literature Review}

\section{SMS Derived Risk}

Derivation Process. To be able to bridge the differences between SMS and public perceived risk, it is first necessary to understand how the SMS risk assessment or perceived risk is derived. The SMS process starts with defining risk.

Stolzer and Goglia (2015) use expected losses mated with the probability of those losses occurring to define risk. Parnell, Driscoll, and Henderson (2011) parallel this definition stating risk is a probabilistic event that when it occurs, causes undesired changes in technical performance, cost or schedule of events. Since risk has been defined, the next major factor to understand is safety risk management (SRM). 
In SMS or system design processes (SDP), SRM is used to identify, assess, and implement risk controls to reduce system variance caused by risks. SRM within the SMS construct uses subject matter experts (SME) to dissect processes, identify hazards, construct a safety risk probability table, build a risk severity table, define levels of risk, and create a risk matrix. SMS perceived risks are those thought to be relevant in the processes used in an organization utilizing SMS. They are derived from risk matrixes based on the overall severity of risks, taking into account probability and severity levels. Risk controls are then implemented to reduce risk level to an acceptable level. It should be noted that acceptable does not mean necessarily reducing the risk level to zero. These perceived risks and associated risk controls are then presented to management in a cost-benefit analysis approach for implementation decisions (Stolzer \& Goglia, 2015). Parnell et al., (2011) describe a similar SMS risk derivation process in identifying, assessing, and mitigating risk. While they use a similar approach, slightly different tools are used such as a risk register, an impact on project table, and a probability impact table. Both risk management processes described are mostly objective in nature, accomplished by experts within an organization, and comprise expert perceived risks or a technological approach (Choi, 2013).

While the SRM process is mostly objective in nature, because of having a defined process, there is still subjectivity introduced into the process, because individuals are involved. Therefore, emotions can influence the risk assessment levels (Slovic, 1997). This inherent subjectivity when assigning levels of severity and probability can prove detrimental when one individual or a group of individuals dominate the SRM process potentially skewing results.

Major Influencing Factors of SMS Derived Risk. In conjunction with understanding how SMS perceived risk is derived, it is also important to grasp the significant influencing factors on the SRM process to have a total understanding of how an organization using SMS derives perceived risk. From a systems engineering perspective in an organization that utilizes SMS, the International Council on Systems Engineering recognizes four risk elements or influencing factors on the process that should be considered when making a system decision. These risk elements include technical, cost, schedule, and programmatic risk. Technical risk is the possibility that the system will not meet a required technical objective or functional requirement. Cost risk is the possibility that the system will exceed the programmed budget. Schedule risk is the possibility that the system will exceed the proposed timeline and associated milestones. Programmatic risk is the possibility of external factors affecting the development and/or deployment of a system 
(Parnell et al., 2011). These four elements used in the derivation process constitute the foundation of the SMS perceived risk assessment.

\section{Public Perceived Risk}

Derivation Process. To be able to mitigate the differences between SMS and public perceived risk to facilitate technology acceptance, one must not only have a good understanding of SMS derivation of perceived risk and influencing factors, but also a good understanding of how public perceived risk is derived and influencing factors. Required knowledge starts with understanding the two levels of perceived risk and cognitive process that the public uses to derive perceived risk.

Dobbie and Brown (2014) distinguish two different levels of perceived risks: expert and layman. While SMS derived perceived risk, otherwise known as expert perceived risk, seems like an all-encompassing risk management approach, being mostly objective in nature, perceived risk derived by the public is mostly based on subjective norms including emotions, and is termed layman perceived risk (Dobbie \& Brown, 2014). Young and Laughery (1994) complement this finding advocating that people use a simple, routine method to derive risk perception that remains identical regardless of technologies being considered. The process is relatively simple with perceived risk derivation occurring in an individual stakeholder's mind without a formal SRM process. A stakeholder is either an organization or individual who has a vested interest in the technology being implemented (Parnell, Driscoll, \&Henderson, 2011). In the context of this paper, stakeholders are the individuals who make up the public realm.

Major Influencing Factors of Public Perceived Risk. Tied to the public perceived risk derivations are the risk elements and associated influencing factors considered in the public stakeholder's cognitive process to derive perceived risk. Thus, understanding the elements and individual influencing factors coupled with the previously discussed public perceived risk derivation process allows an educated gap analysis comparison between SMS and public perceived risk. As discussed, the process the individual public stakeholder uses to derive perceived risk is relatively simple. However, complication ensues when examining the basic elements and the numerous, often subjective, influencing individual factors in an individual's decision process, which at times, may seem irrational.

Risk elements. Six identified elements form the system analysis framework of public perceived risk and include (a) security, (b) financial, (c) physical, (d) social, (e) time, and (f) performance risk. Security risk is the potential threat to an 
individual's security. Financial risk is the likelihood of monetary loss. Physical risk is potential harm to an individual. Social risk refers to potential society disapproval. Time risk is time loss or inconvenience potential. Performance risk is system malfunction potential. These elements are then applied as applicable for the technology studied (Lee, 2009). While a lone individual considering these six elements has little effect on technology acceptance, individuals make up organizations, society groups, industry, and government which can have a profound impact on technology acceptance. While the six elements influence system analysis, individuals vary. Therefore, it is also necessary to study the variables that influence an individual's risk perception formation to have a clear understanding of the public perceived risk influences.

Lack of trust. The first major influencing variable is lack of trust. In public perceived risk studies, lack of trust in the organization using SMS to implement the technology has surfaced as one of the most common influencing factors. There are several contributing factors that researchers have identified as contributing to lack of trust. Whitfield, Rosa, Dan, \& Dietz (2009), Townsend (2013), and Gupta et al. (2012) found that increased trust in government institutions decreased public perceived risk. An additional significant finding was that those individuals with traditional values have more trust in overseeing organizations. Trust is also related to age in that younger people are typically more trusting than older people. One of the biggest mistrust contributors is the perception that the parent organization utilizing SMS is allowing unacceptably high risk (Slovic, 1997). Additionally, in the public's eyes, trust is founded on the organization's sensitivity to their concerns, which can be solely based on emotions, not correct factual information (Lester, 2000). Once the public trust is lost, it is very difficult to regain. Therefore, the focus of the organization using SMS must be on retaining it from the start (Petts, Homan, Breakwell, \& Barnett (2002).

Catastrophic events and mismanagement are other examples of factors that can initiate or deepen an already heightened feeling of public perceived risk or lack of trust. The Three Mile Island power plant partial nuclear meltdown in 1979 is an example of a catastrophic event that had a lasting negative impact on public trust (Whitfield et al., 2009). An example of mismanagement with lasting detrimental effects is that of the weapons plant in Hanford, Washington which in the 1940s and 50 s, released large amounts of radiation contamination into the local ground water which was unknown and undisclosed to the public until recently (Slovic et al., 1991).

Amount of control. Control is another major influencing factor on perceived risk. Lester (2000) states that the basis of the public accepting risk is how 
much control they have over implementing the technology. Further, he advocates that when technology is implemented without public involvement, there is a public perception of a lack of control since they were not involved in the decision-making process. This lack of control results in increased public perceived risk. Nordgren, Pligt, and Harreveld (2007) and Du, Keil, Mathiassen, Shen and Tiwana (2007) agree with Lester concluding that level of control is a major determinant of risk perception level. The more control individuals have, the lower the risk perception. Thus, facilitating technology acceptance through persuasion is easier when the public feels like they have a valid choice of options and are part of the process. Petts et al. (2002) echo these sentiments.

Knowledge and experience. Knowledge of and experience with the technology being also assessed greatly influences public perceived risk. This conclusion is derived from the fact that individual knowledge and experience have a significant impact on individual mental models that use cognitive information processing to form levels of public perceived risk (Dobbie \& Brown, 2014). Sjöberg (1999), and Gupta et al. (2012) found that people with more knowledge about the topic or technology tend to rate risk lower and vice versa. Wildavsky \& Dake (1990) reason that this is true, because with knowledge, people will understand the technology being implemented more. In contrast, Visschers, Meertens, Passchier, and DeVries (2007) stated a "construction-integration" (p, 716) cognitive process derives new information from the text by constructing a rough picture with fill-in information to form a representation. Given that, the mental representation in an individual's mind is only as accurate as the information on which it is based. Related, Whitfield et al. (2009) and Wildavsky and Dake (1990) found people with a lower education level had a higher level of perceived risk. Conversely, Townsend (2013) and Wildavsky and Dake1990) found that people with college degrees rated themselves less at risk than lower educated respondents. Additionally, they found an influencing link between education level and attitude. Significantly, regarding experience, risk perception is derived from an overall experience level that rarely includes experience for the risk in question (Rogers, 1997). Realistic risk perception occurs when people have indirect or direct experience with the risks involved; otherwise, they must rely on other factors (Sjöberg, 2000).

Individual norms/attitudes. Individual norms created by attitudes, individual beliefs and values form the individual public stakeholder's core of perceived risk. Thus, in most instances, differences in risk perception between experts and laypersons can be largely attributed to individual norms (Dobbie \& Brown, 2014). Attitudes form the basis of individual norms and are driven by perceived risk and trust in managing organizations. Once formed, attitudes are 
difficult to change unless individual influencing circumstances change. A good example is nuclear power where public resistance to its usage is high even today. However, even with high public resistance, $42 \%$ of respondents said nuclear power would be acceptable if there were an electricity shortage (Whitfield et al., 2009). A major influencing factor on individual norms is the prevalent attitude a public stakeholder holds. Wildavsky and Dake (1990) identify two prevalent attitudes of risk averseness and risk taking that bias perceived risk. People who are less willing to take risks are defined as risk averse, and those who are more willing to take risks are defined as risk taking.

Individual values. Risk-taking is often a function of the values of an individual. Whitfield et al. (2009), Gupta et al. (2012), and Sjöberg (2000) found a strong correlation between people with traditional values and greater technology acceptance support resulting in less public perceived risk. Those with an unselfish concern for others had less technology acceptance support and a higher public level of perceived risk. Also, with an unfamiliar subject area, Sjöberg (2000) found individuals fall back on norms and; therefore, it can be concluded that risk perception is largely a reflection of individual values. Thus, people rating new technology see mostly good in technology they like and mostly bad things in technology they do not like (Sjöberg, 2000).

Culture theory. A major influence on individual internal beliefs that form public perceived risk is the culture theory. The 15-year-old risk perception culture theory postulates people fall into one of four categories governing individual internal beliefs and risk rating perception: hierarchists, individualists, egalitarians, and fatalists (Sjöberg, 2000). Choi (2013) and Wildavsky \& Dake (1990) further define the categories.

Hiearchists rate factors with the highest impact on social order as most dangerous. Individualists consider factors affecting individual freedom the most dangerous. Sanquist, Mahy \& Morris (2008) echoes the individual freedom aspect. Egalitarians view factors affecting technology and environment as the highest risk. Finally, fatalists are powerless and willing to accept whatever fate risks impose.

Cultural factors. The next major influences on public perceived risk are cultural factors. In conjunction with the four categories, public acceptance, and perception levels vary in different countries due to differing cultural factors (Clothier et. al, 2015; Choi, 2013). As part of the culture influence, social groups form a framework that is a major influence when individual public stakeholders compare their perceived risk ratings to family or other people in the social network (Sjöberg, 2000). Moussaïd (2013) supports this finding that individual risk 
judgments are trended towards individuals in the proximity to the social network which exhibits a strong influence on them.

To be successful in influencing the public's perceived risk level, it is essential to acknowledge the diverse risk perceptions of public stakeholders and to realize that even within the same group, it may difficult to facilitate implementation (Dobbie \& Brown, 2014, La Porte \& Metlay, 1975b). In fact, within the same group, some people may be indifferent and tranquil while others are very upset about perceived risk making it a very dynamic situation to deal with (Sjöberg, 2000).

Seidl, Moser, Stauffacher, \& Krütli (2013) expand on this, listing four possible opinion clusters within a group which includes (a) risk-focused - rates risk high and benefit very low, (b) ambivalent - shows high ratings on both benefit and risk, (c) benefit-focused - rates benefit high and risk very low, and (d) indifferent risk and benefit ratings are both at the moderate level, not feeling strongly either way.

As Moussaïd (2013) argues that there are several possibilities of social groupings including consensus, polarization, and clustering that may be faced by those attempting to persuade the public. Consensus occurs when people agree and support a general opinion. Polarization occurs when two opposed population views emerge and co-exist. Clustering occurs when different groups form with likeminded opinions (Moussaïd, 2013). La Porte and Metlay (1975a) believe that polarization is prevalent in society and; therefore, many technological issues will be in the political arena. In other words, through polarization, people choose a path to support their way of life (Wildavsky \& Dake1990).

Media communication. Media communication is another influencing factor on public perceived risk. The most common types of media communication include pictures and text communication which can be very influential in forming public perceived risk. Pictures affect perceived risk by creating a cognitive image often accompanied times by strong emotions. For example, nuclear war images negatively affected perceived risk regarding nuclear power (Slovic et al., 1991). Clothier et al. (2015) and Sanquist et al. (2008) found that media communication can be very influential in forming perceived risk opinions, especially when there is little subject knowledge. Moussaïd (2013) supports this premise stating that individuals often seek media information to fill knowledge gaps with the amount dictated by existing knowledge. Sjöberg (2000) echoes this but rates it less significant compared to other factors. 
When text media is presented by an organization utilizing SMS, terminology is important, because it often invokes negative connotations, thus increasing perceived risk. For example, nuclear waste disposal terms such as dump, nuclear, or waste have produced negative results. For some people, just the word nuclear invokes negative images of nuclear war with associated effects while invoking a dread or fear factor (Slovic et al., 1991). Also, terminology presented in one location may not be appropriate in another (Petts et al., 2002). Interestingly, Clothier et al. (2015) contrarily found no link between terminology and perceived risk. Simply presenting media in the wrong context can also present problems. The influence of incorrect context was demonstrated by a hypothetical lung cancer study based on choosing between two therapies. When the presentation context of the two therapy choices was changed, results dramatically changed dropping from $44 \%$ to $18 \%$ of those choosing one therapy over the other (Slovic, 1997). This is known as affect heuristic, because if people are upset, they do not think rationally. The other way to describe the effect is how people feel will determine how they think (Greenberg \& Lowrie, 2014; Gupta et al., 2012). Townsend (2013) describes loss aversion bias as another phenomenon framing influence. That is, to rate risk associated with failure higher than associated benefits with success. This bias instills a natural resistance to change that must be overcome (Townsend, 2013). A related significant finding is people associate unknown with known risks to find a relationship based on experience they can understand. Thus, occasionally people use vaguely related or non-related risks to derive opinions. There are four reasons for this that include (a) grasping consequences and severity, (b) to show that other risks have been tolerated, (c) to illustrate there are also benefits to risks, and (d) to show that risks can be resolved (Visschers et al., 2007).

Gender. Gender is the last major influencing factor. Concerning gender, Whitfield et al. (2009) in their nuclear power study, found that women are riskaverse and have a higher level of perceived risk than men. Dobbie and Brown (2014) and Townsend (2013) agree with this assertion.

Age. For age, Joyce, Ferguson, \& Weinstein (2009) in a study of Mars missions determined that school children were more likely to have a higher perceived risk level than adults primarily due to lack of life experiences and young people are more likely to change their values and beliefs than their adult counterparts.

\section{Changing Public Stakeholder Perceived Risk Once an Attitude is formed}

Once formed, public perceived risk changes very slowly even when new information is introduced by an organization using SMS (Dobbie \& Brown, 2014). 
Many attempts to change an already formed attitude failed primarily because the public interrelationship was not handled properly with community acceptance either ignored or diminished. One example was the proposed nuclear waste site at Yucca Mountain, Nevada which was briefly discussed earlier. In this instance, the U.S. Department of Energy (DOE) selected the Yucca Mountain site as the only candidate to store nuclear waste assuming the effort would be successful. However, the people of Nevada convinced the state legislature to pass an assembly bill prohibiting storage of nuclear waste in Nevada. Additionally, a public poll indicated that $80.1 \%$ thought the state "should do all it can to stop the repository" (Slovic, Layman, \& Flynn, 1991, p. 1). The Yucca Mountain incident illustrates the ultimate power of the public's perceived risk of being able to stop technology implementation. Therefore, when technology is being implemented, experts should strive for a mutual understanding of public perceived risks to facilitate acceptance (Lester, 2000).

Public interaction viewed as an opportunity. Public interaction with an organization that utilizes SMS should be viewed as an opportunity rather than an obligation. That is, communication should be focused on influence versus information, and facts not elevated over the public's feelings. Opportunity means going beyond minimal regulation requirements to build community rapport. Focus is needed on persuasion rather than just presenting facts which mean being personal versus technical and listening to subjective reason. Persuasion is driven by emotional, not technical response and involves basic steps (Lester, 2000). This methodology includes (a) understanding public thinking, (b) building credibility by showing concern for citizens, (c) confronting public perceptions and feelings by validating emotions, (d) using one-on-one meetings with the staunchest opponents, (e) tempering the amount of information with the need for information, and (f) involving the public in planning and implementation (Lester, 2000). Zwik (2005) suggest that perceived risk initially derived by the public is often forgotten a short time later. This phenomenon is known as the "switching effect" which reinforces Lester's point of viewing public interaction as an opportunity to change public perceived risk.

Trust restoration. To be able to change public perceived risk, a fundamental requirement is trust restoration. Trust is built on two-way communication, transparency and tight risk controls. This is especially true when an organization that utilizes SMS is implementing new technology as individuals have little subject knowledge regarding complex technical systems (Dobbie \& Brown, 2014). Once trust has been destroyed, long incident-free periods are required to recover. Nuclear examples discussed earlier are good examples of this. 
To address this, trust restoration must be given priority by increasing public involvement in the decision-making process (Slovic et al., 1991).

Public participation. An organization that uses SMS and excludes public participation is often viewed by the public as one attempting to hide critical facts (Lester, 2000). Thus, some researchers advocate more public participation in the SRM process since doing so provides the needed elements of trust and control (Lester, 2000, Slovic et al. 1991). It also allows more democratic decision-making, improves technical assessment relevance, and facilitates public acceptance (Slovic, 1997). However, this approach is not without drawbacks. Public representatives involved in the SRM process for long periods may be out of touch with public wants. Additionally, care must be taken that public representatives do not have a biased personal agenda versus representing the public as a whole. Finally, to be relevant contributors in the SRM process, those involved as public stakeholders require extensive training for unfamiliar jargon and new material (Rogers, Sharp, \& Preece, 2011).

Communication. Communication from an organization using SMS to the public is another major facet of changing public stakeholder perceived risk. There are several communication methods such as broadcast media, printed information, and face-to-face interaction. Clothier et al. (2015), found media to be very influential in individual perceived risk when little is known about the subject. Media timing is critical, though, because once perceived risk attitudes are formed, media has little effect. Therefore, it is imperative that proactive media include a balance of applications, capabilities, risks, and benefits to be effective in perceived risk forming stages (Clothier et al., 2015). The second media form is printed material designed to inform the public about unknown risks ideally spanning the gap between expert risk and public or layman perceived risk similar to an SMS gap analysis (Visschers et al., 2007). Dobbie and Brown, (2014) agree, but instead use the theoretical mental model approach. If media or printed information fails, then using face-to-face communication may be the most effective (Lester, 2000). Ultimately, the risk communication goal should be to garner and incorporate public risk perception information into the risk assessment decisions of the organization utilizing SMS (Rogers, 1997).

\section{Conclusions}

Research has shown, and history has proven, that perceived risk is a significant negative variable affecting technology acceptance success (Clothier, Greer, Greer and Mehta, 2015; Dobbie and Brown, 2014; Gupta, Fischer, and Frewer, 2012; La Porte and Metlay, 1975a; Sjöberg, 2000). More specifically, the 
disparity between SMS and public stakeholder processes coupled with dissimilar influencing factors has, at times, frequently slowed or halted technology implementation. Five other major conclusions can be drawn when comparing SMS and public perceived risk that must be considered as well to ensure acceptance success.

First, perceived risk derived by organizational experts utilizing SMS is fundamentally different than perceived risk derived by individuals. SMS derived risk is mostly objective in nature, because of proven processes with some subjectivity, because individuals are involved. However, individual perceived risk is just the opposite being mostly subjective in nature derived solely by the individual with some objectivity. Because of this, often SMS experts view their process as the ultimate solution, while in the public's eyes, their solution is just as relevant. Thus, an organization using SMS must realize their solution has flaws, and that the public solution is relevant. Therefore, persuasion versus just factual information must be used to garner public support for the technology that is implemented.

Second, when SMS and public influencing elements are compared, there are both similarities and differences. The similar elements between SMS and the public include cost and financial as well as technical and performance. The unique elements to SMS include schedule and programmatic. The elements unique to the public include security, physical, social and time. The unique elements of SMS and those of the public further highlight that there is a fundamental difference between the two risk derivation processes. The significance of this is that the organization utilizing SMS must understand what the elements are for both processes, especially those unique to the public, and address them to win over public support and minimize public perceived risk.

Third, decoding public risk perception is problematic at best, because it is affected not only by the six elements discussed earlier, but other major influencing subjective factors on the individual including (a) lack of trust, (b) amount of control, (c) individual knowledge and experience of the technology being implemented, (d) individual norms, (e) individual values, (f) culture theory, (g) cultural factors, (h) media communication, and (i) gender. It is important to realize from this conclusion that given the numerous major influencing factors coupled with varying individual personality characteristics makes stereotyping all public stakeholders is impossible and influencing public perceived risk very difficult.

Fourth, coupled with these six elements and nine factors, it is essential to understand diverse risk perceptions of stakeholders using the culture theory and 
various social groups during public acceptance to facilitate implementation even within the same group. This is significant because it makes understanding individual public stakeholder perceived risk even more difficult. It also reinforces the fact that just because people are in a group that has a stated position, does not mean assumptions can be made about individual perceived risk levels within the group.

Fifth, changing perceived risk once formed is possible, but very difficult. There is agreement on the methods to accomplish this which includes using persuasion or influence versus presenting just factual information, building trust, and allowing the public to participate in the technology implementation strategy. However, there is disagreement regarding the effectiveness of media communication as an effective influencing factor. Clothier et al. (2015), Moussaïd, M. (2013) and Sanquist et al. (2008) concluded that the media could be a significant influencing factor. Sjöberg (2000) differed concluding it was not as significant as other influencing factors. In conjunction with these tools of influence, being proactive, truthful and understanding to facilitate success are keys to realizing a fundamental shift from an expert technological fact-based approach to one of a subjective perception and social acceptance.

Often, experts gauge public perceived risk as false, concluding that it is based on misunderstanding and ignorance which then creates animosity. The better approach is to realize public perception once formed, while faulty, is valid in the public's eye and that expert risk assessment involves subjectivity and; therefore, is an estimate only. Thus, organizations implementing risk management strategies must take the time to empathize and understand to reshape public concerns given the public trend is becoming more, not less concerned about risk.

It is crucial to have an understanding of SMS derived versus public risk assessment processes and associated influencing factors. Then, if necessary, to apply the knowledge and associated methodology to target public stakeholder perceived risk for either elimination or minimization to facilitate technology implementation.

\section{Recommendations}

This research process highlighted a literature gap in the area of aviation specific technology acceptance studies. Thus, more perceived risk research is needed to fill the aviation related literature gap incorporating major influencing factors. Additionally, there has been much success in applying and validating technology acceptance models such as the Technology Acceptance Model (TAM), 
the Theory of Planned Behavior Model (TPB) and others in information technology. Future research should include an adaptation of one or more of these models for aviation specific technology use. Then, the information in this paper, along with prior research can be used in conjunction with the new model by organizations utilizing SMS to eliminate or mitigate public perceived risk to an acceptable level to enhance aviation technology acceptance. 


\section{References}

Choi, S. (2013). Public perception and acceptability of technological risk: Policy implications for governance. Journal of Convergence Information Technology, 8(13), 605-615. Retrieved from http://www.aicit.org/jcit/home/index.html

Clothier, R. A., Greer, D. A., Greer, D. G., \& Mehta, A. M. (2015). Risk analysis: Risk perception and the public acceptance of drones risk perception and the public acceptance of drones Blackwell Publishing. doi: 10.1111/risa.12330

Dobbie, M. F., \& Brown, R. R. (2014). A framework for understanding risk perception, Explored from the perspective of the eater practitioner. Risk Analysis: An International Journal, 34(2), 294-308. doi: 10.1111/risa.12100

Du, S., Keil, M., Mathiassen, L., Shen, Y., \& Tiwana, A. (2007). Attentionshaping tools, expertise, and perceived control in IT project risk assessment. Decision Support Systems, 43(1), 269-283. doi:10.1016/j.dss.2006.10.002

Greenberg, M., \& Lowrie, K. (2014). Paul Slovic: Risk perceptions and affect. Risk Analysis: An International Journal, 34(2), 206-209. doi: 10.111/risa.12163

Gupta, N., Fischer, R.H., \& Frewer, L.J. (2012). Socio-psychological determinants of public acceptance of technologies: A review. Public Understanding of Science, 21(7), 782-795. doi: 10.1177/0963662510 392485

Hunter, R. (2001, 09). The public perception of risk. Australasian Science, 22, 3032. Retrieved from http://www.australasianscience.com.au

Joyce, S., Ferguson, C., \& Weinstein, P. (2009). Public support for Mars missions: The importance of informing the next generation. Acta Astronautica, 64(7-8), 718-723. doi:10.1016/j.actaastro.2008.12.014

LaPorte, T., and D. Metlay. (1975a). Public attitudes toward present and future technologies. Social Studies of Science 5, 373-398. Retrieved from http://www.4sonline.org/ 
LaPorte, T., \& Metlay, D. (1975b). They watch and wonder. Public attitudes toward advanced technology final report. (Report No. NASA-CR149673). Retrieved from National Aeronautics and Space website http://ntrs.nasa.gov/archive/nasa/casi.ntrs.nasa.gov/19770012010.pdf

Lawrence, H. (2014). Aviation and the role of government. (3rd ed.) Dubuque, IA: Kendall Hunt.

Lee, M. (2009). Factors influencing the adoption of internet banking: An integration of TAM and TPB with perceived risk and perceived benefit. Electronic Commerce Research and Applications, 8(3), 130-141. doi: 10.1016/j.elerap.2008.11.006

Lester, M. (2000). Communicate risk effectively. Chemical Engineering Progress, 96(6), 79. Retrieved from http://www.aiche.org/resources/publications/cep

Moussaïd, M. (2013). Opinion formation and the collective dynamics of risk perception. PLoS One, 8(12) doi:10.1371/journal.pone.0084592

Nordgren, L. F., Pligt, v. d., J, \& Harreveld, v., F. (2007). Unpacking perceived control in risk perception: The mediating role of anticipated regret. Journal of Behavioral Decision Making, 20(5), 533-544. doi:10.1002/bdm.565

Petts, J., Homan, J, Breakwell, G., \& Barnett, J. (2002) Understanding public perception of risk: report of an environment agency workshop. Project Record: P5-040/PR1. Retrieved from https://www.gov.uk/government/publications/understanding-publicperception-of-risk-report-of-an-environment-agency-workshop

Parnell, G. S., Driscoll, P. J., \& Henderson, D. L. (2011). Decision making in systems engineering and management. (2nd ed.). Hoboken, NJ: Wiley and Sons.

Rogers, G. O. (1997). The dynamics of risk perception: How does perceived risk respond to risk events? Risk Analysis, 17(6), 745-757. doi: 10.1111/j.15396924.1997.tb01280.x 
Rogers, Y., Sharp, H., \& Preece, J. (2011). Interaction design: beyond humancomputer interaction. (3rd ed.). Hoboken, NJ: John Wiley and Sons.

Sanquist, T. F., Mahy, H., \& Morris, F. (2008). An exploratory risk perception study of attitudes toward homeland security systems. Risk Analysis: An International Journal, 28(4), 1125-1133. doi: 10.1111/j.15396924.2008.01069.x

Seidl, R., Moser, C., Stauffacher, M., \& Krütli, P. (2013). Perceived risk and benefit of nuclear waste repositories: Four opinion clusters. Risk Analysis, 33(6), 1038-1048. doi:10.1111/j.1539-6924.2012.01897.x

Slovic, P., Layman, M., \& Flynn, J. H. (1991). Risk perception, trust, and nuclear waste: Lessons from Yucca Mountain. Environment, 33(3), 6. doi: 10.1080/00139157.1991.9931375

Slovic, P. (1997). Public perception of risk. Journal of Environmental Health, 59(9), 22-23+. Retrieved from http://www.neha.org/publications/journalenvironmental-health

Sjöberg, L. (1999). Risk perception by the public and by experts: A dilemma in risk management. Human Ecology Review, 6(2), 1-9. Retrieved from http://www.humanecologyreview.org/pastissues/her62/62sjoberg.pdf

Sjöberg, L. (2000). Factors in risk perception. Risk analysis, 20(1), 1-12. doi: $10.1111 / 0272-4332.00001$

Stolzer, A. J., \& Goglia, J. J. (2015). Safety management systems in aviation. (2nd ed.). Burlington, VT: Ashgate.

Townsend, W. (2013). Innovation and the perception of risk in the public sector. International Journal of Organizational Innovation, 5(3), 21-34. Retrieved from http://ijoi-online.org/

Visschers, V. M., Meertens, R. M., Passchier, W. F., \& DeVries, N. K. (2007). How does the general public evaluate risk information? The impact of associations with other risks. Risk Analysis: An International Journal, 27(3), 715-727. doi: 10.1111/j.1539-6924.2007.00915.x 
Young, S. L., \& Laughery, K. R., (1994). Components of perceived risk: A reconciliation of previous findings. Proceedings of the Human Factors and Ergonomics Society Annual Meeting, 38, 888-892. doi: $10.1177 / 154193129403801420$

Warwick, G. (2013). Harnessing autonomy. Aviation Week \& Space Technology, 175(27), 48. Retrieved from http://aviationweek.com/aviation-weekspace-technology

Whitfield, S. C., Rosa, E. A., Dan, A., \& Dietz, T. (2009). The future of nuclear power: Value orientations and risk perception. Risk Analysis: An International Journal, 29(3), 425-437. doi: 10.1111/j.15396924.2008.01155.x

Wildavsky, A., \& Dake, K. (1990). Theories of risk perception: Who fears what and why? Daedalus, 119(4), 41. Retrieved from http://www.jstor.org/ stable/20025337

Zwick, M. M. (2005). Risk as perceived by the German public: Pervasive risks and "switching" risks. Journal of Risk Research, 8(6), 481-498. doi:10.1080/13669870500064150 